\title{
Strongly Nonlinear Differential Equations with Carlitz Derivatives over a Function Field
}

\author{
ANATOLY N. KOCHUBEI* \\ Institute of Mathematics, National Academy \\ of Sciences of Ukraine, Tereshchenkivska 3, Kiev, 01601 Ukraine
}

\begin{abstract}
In earlier papers the author studied some classes of equations with Carlitz derivatives for $\mathbb{F}_{q}$-linear functions, which are the natural function field counterparts of linear ordinary differential equations. Here we consider equations containing self-compositions $u \circ u \circ \cdots \circ u$ of the unknown function. As an algebraic background, imbeddings of the composition ring of $\mathbb{F}_{q}$-linear holomorphic functions into skew fields are considered.
\end{abstract}

\section{INTRODUCTION}

Let $K$ be the set of formal Laurent series $t=\sum_{j=N}^{\infty} \xi_{j} x^{j}$ with coefficients $\xi_{j}$ from the Galois field $\mathbb{F}_{q}, \xi_{N} \neq 0$ if $t \neq 0, q=p^{v}, v \in \mathbf{Z}_{+}$, where $p$ is a prime number. It is well known that $K$ is a locally compact field of characteristic $p$, with natural operations over power series, and the topology given by the absolute value $|t|=q^{-N},|0|=0$. The element $x$ is a prime element of $K$. Any non-discrete locally compact field of characteristic $p$ is isomorphic to such $K$. Below we denote by $\bar{K}_{c}$ the completion of an algebraic closure $\bar{K}$ of $K$. The absolute value $|\cdot|$ can be extended in a unique way onto $\bar{K}_{c}$.

An important class of functions playing a significant part in the analysis over $\bar{K}_{c}$ is the class of $\mathbb{F}_{q}$-linear functions. A function $f$ defined on a $\mathbb{F}_{q}$-subspace $K_{0}$ of $K$ (or $\bar{K}_{c}$ ), with values in $\bar{K}_{c}$, is called $\mathbb{F}_{q}$-linear if $f\left(t_{1}+t_{2}\right)=f\left(t_{1}\right)+f\left(t_{2}\right)$ and $f(\alpha t)=\alpha f(t)$ for any $t, t_{1}, t_{2} \in K_{0}$, $\alpha \in \mathbb{F}_{q}$. A typical example is a $\mathbb{F}_{q}$-linear polynomial $\sum c_{k} t^{q^{k}}$ or, more generally, a power series $\sum_{k=0}^{\infty} c_{k} t^{q^{k}}$, where $c_{k} \in \bar{K}_{c}$ and $\left|c_{k}\right| \leq C^{q^{k}}$, convergent on a neighbourhood of the origin.

In the theory of differential equations over $K$ initiated in [7, 8] (which deals also with some non-analytic $\mathbb{F}_{q}$-linear functions) the role of a derivative is played by the operator

$$
d=\sqrt[q]{\circ} \Delta, \quad(\Delta u)(t)=u(x t)-x u(t),
$$

introduced by Carlitz [1] and used subsequently in various problems of analysis in positive characteristic [2, 3, 6, 11, 12].

*This research was supported in part by CRDF under Grants UM1-2421-KV-02 and UM1-2567-OD-03. 
The differential equations considered so far were analogs of linear ordinary differential equations, though the operator $d$ is only $\mathbb{F}_{q}$-linear and the meaning of a polynomial coefficient in the function field case is not a usual multiplication by a polynomial, but the action of a polynomial in the $\mathbb{F}_{q}$-linear operator $\tau, \tau u=u^{q}$. Note that $\mathbb{F}_{q}$-linear polynomials form a ring with respect to the composition $u \circ v$ (the usual multiplication violates the $\mathbb{F}_{q}$-linearity), so that natural classes of equations with stronger nonlinearities must contain expressions like $u \circ u$ or, more generally, $u \circ u \cdots \circ u$. An investigation of such "strongly nonlinear" Carlitz differential equations is the main aim of this paper.

However we have to begin with algebraic preliminaries of some independent interest (so that not all the results are used in the subsequent sections) regarding the ring $\mathcal{R}_{K}$ of locally convergent $\mathbb{F}_{q}$-linear holomorphic functions. The ring is non-commutative, and the algebraic structures related to strongly nonlinear Carlitz differential equations are much more complicated than their classical counterparts. So far their understanding is only at its initial stage. Here we show that $\mathcal{R}_{K}$ is imbedded into a skew field of $\mathbb{F}_{q}$-linear "meromorphic" series containing terms like $t^{q^{-k}}$. Note that a deep investigation of bi-infinite series of this kind convergent on the whole of $\bar{K}_{c}$ has been carried out by Poonen [10. We also prove an appropriate version of the implicit function theorem.

After the above preparations we consider general strongly nonlinear first order $\mathbb{F}_{q}$-linear differential equations (resolved with respect to the derivative of the unknown function) and prove an analog of the classical Cauchy theorem on the existence and uniqueness of a local holomorphic solution of the Cauchy problem. In our case the classical majorant approach (see e.g. [5]) does not work, and the convergence is proved by direct estimates. We also consider a class of Riccati-type equations possessing $\mathbb{F}_{q}$-linear solutions which are meromorphic in the above sense.

\section{Skew fields of $\mathbb{F}_{q}$-linear power series}

Let $\mathcal{R}_{K}$ be the set of all formal power series $a=\sum_{k=0}^{\infty} a_{k} t^{t^{k}}$ where $a_{k} \in K,\left|a_{k}\right| \leq A^{q^{k}}$, and $A$ is a positive constant depending on $a$. In fact each series $a=a(t)$ from $\mathcal{R}_{K}$ converges on a neighbourhood of the origin in $K$ (and $\bar{K}_{c}$ ).

$\mathcal{R}_{K}$ is a ring with respect to the termwise addition and the composition

$$
a \circ b=\sum_{l=0}^{\infty}\left(\sum_{n=0}^{l} a_{n} b_{l-n}^{q^{n}}\right) t^{q^{l}}, \quad b=\sum_{k=0}^{\infty} b_{k} t^{q^{k}},
$$

as the operation of multiplication. Indeed, if $\left|b_{k}\right| \leq B^{q^{k}}$, then, by the ultra-metric property of the absolute value,

$$
\left|\sum_{n=0}^{l} a_{n} b_{l-n}^{q^{n}}\right| \leq \max _{0 \leq n \leq l} A^{q^{n}}\left(B^{q^{l-n}}\right)^{q^{n}} \leq C^{q^{l}}
$$

where $C=B \max (A, 1)$. The unit element in $\mathcal{R}_{K}$ is $a(t)=t$. It is easy to check that $\mathcal{R}_{K}$ has no zero divisors. 
If $a \in \mathcal{R}_{K}, a=\sum_{k=0}^{\infty} a_{k} t^{q^{k}}$, is such that $\left|a_{0}\right| \leq 1$ and $\left|a_{k}\right| \leq A^{q^{k}},|A| \geq 1$, for all $k$, then we may write

$$
\left|a_{k}\right| \leq A_{1}^{q^{k}-1}, \quad k=0,1,2, \ldots
$$

if we take $A_{1} \geq A^{q^{k} /\left(q^{k}-1\right)}$ for all $k \geq 1$. If also $b=\sum_{k=0}^{\infty} b_{k} t^{q^{k}},\left|b_{k}\right| \leq B_{1}^{q^{k}-1}, B_{1} \geq 1$, then for $a \circ b=\sum_{l=0}^{\infty} c_{l} t^{q^{l}}$ we have

$$
\left|c_{l}\right| \leq \max _{i+j=l} A_{1}^{q^{i}-1}\left(B_{1}^{q^{j}-1}\right)^{q^{i}} \leq C_{1}^{q^{l}-1}
$$

where $C_{1}=\max \left(A_{1}, B_{1}\right)$. In particular, in this case the coefficients of the series for $a^{n}$ (the composition power) satisfy an estimate of this kind, with a constant independent of $n$.

Proposition 1. The ring $\mathcal{R}_{K}$ is a left Ore ring, thus it possesses a classical ring of fractions.

Proof. By Ore's theorem (see [4]) it suffices to show that for any elements $a, b \in \mathcal{R}_{K}$ there exist such elements $a^{\prime}, b^{\prime} \in \mathcal{R}_{K}$ that $b^{\prime} \neq 0$ and

$$
a^{\prime} \circ b=b^{\prime} \circ a \text {. }
$$

We may assume that $a \neq 0$,

$$
a=\sum_{k=m}^{\infty} a_{k} t^{q^{k}}, \quad b=\sum_{k=l}^{\infty} b_{k} t^{q^{k}}
$$

$m, l \geq 0, a_{m} \neq 0, b_{l} \neq 0$.

Without restricting generality we may assume that $l=m$ (if we prove (1) for this case and if, for example, $l<m$, we set $b_{1}=t^{q^{m-l}} \circ b$, find $a^{\prime \prime}, b^{\prime}$ in such a way that $a^{\prime \prime} \circ b_{1}=b^{\prime} \circ a$, and then set $\left.a^{\prime}=a^{\prime \prime} \circ t^{q^{m-l}}\right)$, and that $a_{l}=b_{l}=\alpha$, so that

$$
a=\alpha t^{q^{l}}+\sum_{k=l+1}^{\infty} a_{k} t^{q^{k}}, \quad b=\alpha t^{q^{l}}+\sum_{k=l+1}^{\infty} b_{k} t^{q^{k}}
$$

$\alpha \neq 0$.

We seek $a^{\prime}, b^{\prime}$ in the form

$$
a^{\prime}=\sum_{j=l}^{\infty} a_{j}^{\prime} t^{q^{j}}, \quad b^{\prime}=\sum_{j=l}^{\infty} b_{j}^{\prime} t^{q^{j}}
$$

The coefficients $a_{j}^{\prime}, b_{j}^{\prime}$ can be defined inductively. Set $a_{l}^{\prime}=b_{l}^{\prime}=1$. If $a_{j}^{\prime}$, $b_{j}^{\prime}$ have been determined for $l \leq j \leq k-1$, then $a_{k}^{\prime}, b_{k}^{\prime}$ are determined from the equality of the $(k+l)$-th terms of the composition products:

$$
a_{k}^{\prime} \alpha^{q^{k}}+\sum_{\substack{i+j=k+l \\ j \neq l}} a_{i}^{\prime} b_{j}^{q^{i}}=b_{k}^{\prime} \alpha^{q^{k}}+\sum_{\substack{i+j=k+l \\ j \neq l}} b_{i}^{\prime} a_{j}^{q^{i}}
$$


(the above sums do not contain non-trivial terms with $a_{i}^{\prime}, b_{i}^{\prime}, i \geq k$, since $a_{j}=b_{j}=0$ for $j<l$ ).

In particular, we may set $b_{k}^{\prime}=0$,

$$
a_{k}^{\prime}=\alpha^{-q^{k}}\left\{\sum_{\substack{i+j=k+l \\ i<k, j \neq l}}\left(a_{i}^{\prime} b_{j}^{q^{i}}-b_{i}^{\prime} a_{j}^{q^{i}}\right)\right\} .
$$

If this choice is made for each $k \geq l+1$, then we have $b_{i}^{\prime}=0$ for every $i \geq l+1$, so that

$$
a_{k}^{\prime}=\alpha^{-q^{k}} \sum_{\substack{i+j=k+l \\ i<k, j \neq l}} a_{i}^{\prime} b_{j}^{q^{i}} .
$$

Denote $C_{1}=|\alpha|^{-1}$. We have $\left|b_{j}\right| \leq C_{2}^{q^{j}}$ for all $j$. Denote, further, $C_{3}=\max \left(1, C_{1}, C_{2}\right)$, $C_{4}=C_{3}^{q^{l+2}}$. Let us prove that

$$
\left|a_{k}^{\prime}\right| \leq C_{4}^{q^{k}}
$$

Suppose that $\left|a_{i}^{\prime}\right| \leq C_{4}^{q^{i}}$ for all $i, l \leq i \leq k-1$ (this is obvious for $i=1$, since $a_{l}^{\prime}=1$ ). By $(2)$,

$$
\begin{aligned}
\left|a_{k}^{\prime}\right| \leq C_{1}^{q^{k}} \max _{\substack{i+j=k+l \\
i<k, j \neq l}} C_{4}^{q^{i}} C_{2}^{q^{i+j}} \leq C_{1}^{q^{k}} C_{4}^{q^{k-1}} & C_{2}^{q^{k+l}} \\
& \leq C_{3}^{q^{k}+q^{k+l+1}+q^{k+l}}=C_{3}^{\left(1+q^{l}+q^{l+1}\right) q^{k}} \leq\left(C_{3}^{q^{l+2}}\right)^{q^{k}}=C_{4}^{q^{k}},
\end{aligned}
$$

as desired. Thus $a^{\prime} \in \mathcal{R}_{K}$.

Every non-zero element of $\mathcal{R}_{K}$ is invertible in the ring of fractions $\mathcal{A}_{K}$, which is actually a skew field consisting of formal fractions $c^{-1} d, c, d \in \mathcal{R}_{K}$.

Proposition 2. Each element $a=c^{-1} d \in \mathcal{A}_{K}$ can be represented in the form $a=t^{q^{-m}} a^{\prime}$ where $t^{q^{-m}}$ is the inverse of $t^{q^{m}}, a^{\prime} \in \mathcal{R}_{K}$.

Proof. It is sufficient to prove that any non-zero element $c \in \mathcal{R}_{K}$ can be written as $c=c^{\prime} \circ t^{q^{m}}$ where $c$ is invertible in $\mathcal{R}_{K}$.

Let $c=\sum_{k=m}^{\infty} c_{k} t^{q^{k}}, c_{m} \neq 0,\left|c_{k}\right| \leq C^{q^{k}}$. Then

$$
c=c_{m}\left(t+\sum_{l=1}^{\infty} c_{m}^{-1} c_{m+l} t^{q^{l}}\right) \circ t^{q^{m}}
$$

where $\left|c_{m}^{-1} c_{m+l}\right| \leq C_{1}^{q^{l}-1}$ for all $l \geq 1$, if $C_{1}$ is sufficiently large. Denote

$$
w=\sum_{l=1}^{\infty} c_{m}^{-1} c_{m+l} t^{t^{l}}, \quad c^{\prime}=c_{m}(t+w) .
$$

The series $(t+w)^{-1}=\sum_{n=0}^{\infty}(-1)^{n} w^{n}$ converges in the standard non-Archimedean topology of formal power series (see [9], Sect. 19.7) because the formal power series for $w^{n}$ begins 
from the term with $t^{q^{m}}$; recall that $w^{n}$ is the composition power, and $t$ is the unit element. Moreover, $w^{n}=\sum_{j=n}^{\infty} a_{j}^{(n)} t^{q^{j}}$ where $\left|a_{j}^{(n)}\right| \leq C_{1}^{q^{j}-1}$ for all $\mathrm{j}$, with the same constant independent of $n$. Using the ultra-metric inequality we find that the coefficients of the formal power series $(t+w)^{-1}=\sum_{j=0}^{\infty} a_{j} t^{q^{j}}$ (each of them is, up to a sign, a finite sum of the coefficients $a_{j}^{(n)}$ ) satisfy the same estimate. Therefore $\left(c^{\prime}\right)^{-1} \in \mathcal{R}_{K}$.

The skew field of fractions $\mathcal{A}_{K}$ can be imbedded into wider skew fields where operations are more explicit. Let $K_{\text {perf }}$ be the perfection of the field $K$. Denote by $\mathcal{A}_{K_{\text {perf }}}^{\infty}$ the composition ring of $\mathbb{F}_{q}$-linear formal Laurent series $a=\sum_{k=m}^{\infty} a_{k} t^{q^{k}}, m \in \mathbb{Z}, a_{k} \in K_{\text {perf }}, a_{m} \neq 0$ (if $a \neq 0$ ). Since $\tau$ is an automorphism of $K_{\text {perf }}, \mathcal{A}_{K_{\text {perf }}}^{\infty}$ is a special case of the well-known ring of twisted Laurent series [9]. Therefore $\mathcal{A}_{K_{\text {perf }}}^{\infty}$ is a skew field.

Let $\mathcal{A}_{K_{\text {perf }}}$ be a subring of $\mathcal{A}_{K_{\text {perf }}}^{\infty}$ consisting of formal series with $\left|a_{k}\right| \leq A^{q^{k}}$ for all $k \geq 0$. Just as in the proof of Proposition 2, we show that $\mathcal{A}_{K_{\text {perf }}}$ is actually a skew field. Its elements can be written in the form $t^{q^{-m}} \circ c$ where $c$ is an invertible element of the ring $\mathcal{R}_{K_{\text {perf }}} \in \mathcal{A}_{K_{\text {perf }}}$ of formal power series $\sum_{k=0}^{\infty} a_{k} t^{q^{k}}$. In contrast to the case of the skew field $\mathcal{A}_{K}$, in $\mathcal{A}_{K_{\text {perf }}}$ the multiplication of $t^{q^{-m}}$ by $c$ is indeed the composition of (locally defined) functions, so that $\mathcal{A}_{K_{\text {perf }}}$ consists of fractional power series understood in the classical sense.

Of course, $\mathcal{A}_{K_{\text {perf }}}$ can be extended further, by considering $\bar{K}$ or $\bar{K}_{c}$ instead of $K_{\text {perf. }}$ The above reasoning carries over to these cases (we can also consider the ring $\mathcal{R}_{\bar{K}_{c}}$ of locally convergent $\mathbb{F}_{q}$-linear power series as the initial ring). In each of them the presence of a fractional composition factor $t^{q^{-m}}$ is a $\mathbb{F}_{q^{-}}$linear counterpart of a pole of the order $m$.

\section{Recurrent relations}

In our investigations of strongly nonlinear equations and implicit functions we encounter recurrent relations of the same form

$$
c_{i+1}=\mu_{i} \sum_{\substack{j+l=i \\ l \neq 0}} \sum_{k=1}^{\infty} B_{j k l}\left(\sum_{n_{1}+\cdots+n_{k}=l} c_{n_{1}} c_{n_{2}}^{q^{n_{1}}} \cdots c_{n_{k}}^{q^{n_{1}+\cdots+n_{k-1}}}\right)^{q^{j+\lambda}}+a_{i}, \quad i=1,2, \ldots,
$$

(here and below $n_{1}, \ldots, n_{k} \geq 1$ in the internal sum), with coefficients from $\bar{K}_{c}$, such that $\left|\mu_{i}\right| \leq M, M>0,\left|B_{j k l}\right| \leq B^{k q^{j}}, B \geq 1,\left|a_{i}\right| \leq M$ for all $i, j, k, l$; the number $\lambda$ is either equal to 1 , or $\lambda=0$, and in that case $\left|B_{01 l}\right| \leq 1$.

Proposition 3. For an arbitrary element $c_{1} \in \bar{K}_{c}$, the sequence determined by the relation (3) satisfies the estimate $\left|c_{n}\right| \leq C^{q^{n}}, n=1,2, \ldots$, with some constant $C \geq 1$. 
Proof. Set $c_{n}=\sigma d_{n},|\sigma|<1, n=1,2, \ldots$, and substitute this into (3). We have

$$
\begin{array}{r}
d_{i+1}=\mu_{i}\left\{\sum_{\substack{j+l=i \\
l \neq 0}} \sum_{k=1}^{\infty} B_{j k l} \sum_{n_{1}+\cdots+n_{k}=l} \sigma^{\left(1+q^{n_{1}}+\cdots+q^{n_{1}+\cdots+n_{k-1}}\right)^{q^{j+\lambda}}-1}\right. \\
\left.\times\left(d_{n_{1}} d_{n_{2}}^{q^{n_{1}}} \cdots d_{n_{k}}^{q^{n_{1}+\cdots+n_{k-1}}}\right)^{q^{j+\lambda}}\right\}+\sigma^{-1} a_{i} .
\end{array}
$$

Here

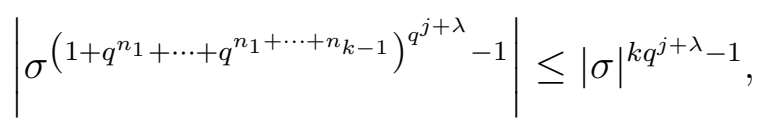

and (under our assumptions) choosing such $\sigma$ that $|\sigma|$ is small enough we reduce (3) to the relation

$$
d_{i+1}=\mu_{i} \sum_{\substack{j+l=i \\ l \neq 0}} \sum_{k=1}^{\infty} b_{j k l} \sum_{n_{1}+\cdots+n_{k}=l}\left(d_{n_{1}} d_{n_{2}}^{q^{n_{1}}} \cdots d_{n_{k}}^{q^{n_{1}+\cdots+n_{k-1}}}\right)^{q^{j+\lambda}}+\sigma^{-1} a_{i}, \quad i=1,2, \ldots
$$

where $\left|b_{j k l}\right| \leq 1$.

It follows from (4) that

$\left|d_{i+1}\right| \leq M \max _{\substack{j+l=i \\ l \neq 0}} \sup _{\substack{ \\l \geq 1}} \max _{n_{1}+\cdots+n_{k}=l} \max \left\{\left(\left|d_{n_{1}}\right| \cdot\left|d_{n_{2}}^{q_{1}}\right| \cdots\left|d_{n_{k}}\right|^{q^{n_{1}+\cdots+n_{k-1}}}\right)^{q^{j+\lambda}}, M^{-1}\left|\sigma^{-1} a_{i}\right|\right\}$.

Let $B=\max \left\{1, M,\left|d_{1}\right|, M^{-1} \sup _{i}\left|\sigma^{-1} a_{i}\right|\right\}$. Let us show that

$$
\left|d_{n}\right| \leq B^{q^{n-1}+q^{n-2}+\cdots+1}, \quad n=1,2, \ldots
$$

This is obvious for $n=1$. Suppose that we have proved (5) for $n \leq i$. Then

$$
\begin{array}{r}
\left|d_{i+1}\right| \leq M \max _{j+l=i} \sup _{k \geq 1} \max _{n_{1}+\cdots+n_{k}=l}\left(B^{q^{n_{1}-1}+q^{n_{1}-2}+\cdots+1} \cdot B^{q^{n_{1}+n_{2}-1}+q^{n_{1}+n_{2}-2}+\cdots+q^{n_{1}}} \cdots\right. \\
\left.\times \cdots B^{q^{n_{1}+\cdots+n_{k-1}+n_{k}-1}+q^{n_{1}+\cdots+n_{k-1}+n_{k}-2}+\cdots+1}+q^{n_{1}+\cdots+n_{k-1}}\right)^{q^{j+1}} \leq M \max _{j+l=i} B^{q^{j+l}+\cdots+q^{j+1}} \\
\leq B \cdot B^{q^{i}+q^{i-1}+\cdots+q}=B^{q^{i}+q^{i-1}+\cdots+1},
\end{array}
$$

and we have proved (5). Therefore

$$
\left|c_{n}\right| \leq|\sigma| B^{\frac{q^{n}-1}{q-1}} \leq C^{q^{n}}
$$

for some $C$, as desired. 


\section{Implicit functions of algebraic type}

In this section we look for $\mathbb{F}_{q}$-linear locally holomorphic solutions of equations of the form

$$
P_{0}(t)+P_{1}(t) \circ z+P_{2}(t) \circ(z \circ z)+\cdots+P_{N}(t) \circ \underbrace{(z \circ z \circ \cdots \circ z)}_{N}=0
$$

where $P_{0}, P_{1}, \ldots P_{N} \in \mathcal{R}_{\bar{K}_{c}}$. Suppose that the coefficient $P_{k}(t)=\sum_{j \geq 0} a_{j k} t^{q^{j}}$ is such that $a_{00}=0$, $a_{01} \neq 0$; these assumptions are similar to the ones guaranteeing the existence and uniqueness of a solution in the classical complex analysis. Then (see Sect. 2) $P_{1}$ is invertible in $\mathcal{R}_{\bar{K}_{c}}$, and we can rewrite $(6)$ in the form

$$
z+Q_{2}(t) \circ(z \circ z)+\cdots+Q_{N}(t) \circ \underbrace{(z \circ z \circ \cdots \circ z)}_{N}=Q_{0}(t)
$$

where $Q_{0}, Q_{2}, \ldots, Q_{N} \in \mathcal{R}_{\bar{K}_{c}}$, that is

$$
Q_{k}(t)=\sum_{j=0}^{\infty} b_{j k} t^{q^{j}}, \quad\left|b_{j k}\right| \leq B_{k}^{q^{j}}
$$

for some constants $B_{k}>0$, and $b_{00}=0$.

Proposition 4. The equation (6) has a unique solution $z \in \mathcal{R}_{\bar{K}_{c}}$ satisfying the "initial condition"

$$
\frac{z(t)}{t} \longrightarrow 0, \quad t \rightarrow 0
$$

Proof. Let us look for a solution of the transformed equation (7), of the form

$$
z(t)=\sum_{i=1}^{\infty} c_{i} t^{q^{i}}, \quad c_{i} \in \bar{K}_{c}
$$

our initial condition is automatically satisfied for a function (8).

Substituting (8) into (7) we come to the system of equalities

$$
c_{i}=-\sum_{k=2}^{N} \sum_{\substack{j+l=i \\ j \geq 0, l \geq 1}} b_{j k}\left(\sum_{\substack{n_{1}+\cdots+n_{k}=l \\ n_{j} \geq 1}} c_{n_{1}} c_{n_{2}}^{q^{n_{1}}} \cdots c_{n_{k}}^{q^{n_{1}+\cdots+n_{k-1}}}\right)^{q^{j}}+b_{i 0}, \quad i \geq 1 .
$$

In each of them the right-hand side depends only on $c_{1}, \ldots, c_{i-1}$, so that the relations $(9)$ determine the coefficients of a solution (8) uniquely. By Proposition $3, z \in \mathcal{R}_{\bar{K}_{c}}$.

More generally, let

$$
P_{1}(t)=\sum_{j \geq \nu} a_{j 1} t^{q^{j}}, \quad \nu \geq 0, \quad a_{\nu 1} \neq 0 .
$$

Then the equation (6) has a unique solution in $\mathcal{R}_{\bar{K}_{c}}$, of the form

$$
z(t)=\sum_{i=\nu+1}^{\infty} c_{i} t^{q^{i}}, \quad c_{i} \in \bar{K}_{c}
$$

The proof is similar. 


\section{Equations with Carlitz derivatives}

Let us consider the equation

$$
d z(t)=\sum_{j=0}^{\infty} \sum_{k=1}^{\infty} a_{j k} \tau^{j} \underbrace{(z \circ z \circ \cdots \circ z)}_{k}(t)+\sum_{j=0}^{\infty} a_{j 0} t^{q^{j}}
$$

where $a_{j k} \in \bar{K}_{c},\left|a_{j k}\right| \leq A^{k q^{j}}(k \geq 1),\left|a_{j 0}\right| \leq A^{q^{j}}, A \geq 1$. We look for a solution in the class of $\mathbb{F}_{q^{-}}$-linear locally holomorphic functions of the form

$$
z(t)=\sum_{k=1}^{\infty} c_{k} t^{q^{k}}, \quad c_{k} \in \bar{K}_{c}
$$

thus assuming the initial condition $t^{-1} z(t) \rightarrow 0$, as $t \rightarrow 0$.

Theorem 1. A solution (11) of the equation (10) exists with a non-zero radius of convergence, and is unique.

Proof. We may assume that

$$
\left|a_{j 0}\right| \leq 1, \quad a_{j 0} \rightarrow 0, \quad \text { as } j \rightarrow \infty .
$$

Indeed, if that is not satisfied, we can perform a time change $t=\gamma t_{1}$ obtaining an equation of the same form, but with the coefficients $a_{j 0} \gamma^{q^{j}}$ instead of $a_{j 0}$, and it remains to choose $\gamma$ with $|\gamma|$ small enough. Note that, in contrast with the case of the usual derivatives, the operator $d$ commutes with the above time change.

Assuming (12) we substitute (11) into (10) using the fact that $d\left(c_{k} t^{q^{k}}\right)=c_{k}^{1 / q}[k]^{1 / q} t^{q^{k-1}}$, $k \geq 1$, where $[k]=x^{q^{k}}-x$. Comparing the coefficients we come to the recursion

$$
c_{i+1}=[i+1]^{-1} \sum_{\substack{j+l=i \\ j \geq 0, l \geq 1}} \sum_{k=1}^{\infty} a_{j k}^{q}\left(\sum_{n_{1}+\cdots+n_{k}=l} c_{n_{1}} c_{n_{2}}^{q^{n_{1}}} \cdots c_{n_{k}}^{q^{n_{1}+\cdots+n_{k-1}}}\right)^{q^{j+1}}+a_{i 0}, \quad i \geq 1
$$

where $c_{1}=[1]^{-1} a_{00}^{q}$. This already shows the uniqueness of a solution. The fact that $\left|c_{i}\right| \leq C^{q^{i}}$ for some $C$ follows from Proposition 3.

Using Proposition 4 we can easily reduce to the form (10) some classes of equations given in the form not resolved with respect to $d z$.

As in the classical case of equations over $\mathbb{C}$ (see [5]), some of equations (10) can have also non-holomorphic solutions, in particular those which are meromorphic in the sense of Sect. 2. As an example, we consider Riccati-type equations

$$
d y(t)=\lambda(y \circ y)(t)+(P(\tau) y)(t)+R(t)
$$

where $\lambda \in \bar{K}_{c}, 0<|\lambda| \leq q^{-1 / q^{2}}$

$$
(P(\tau) y)(t)=\sum_{k=1}^{\infty} p_{k} y^{q^{k}}(t), \quad R(t)=\sum_{k=0}^{\infty} r_{k} t^{q^{k}}
$$

$p_{k}, r_{k} \in \bar{K}_{c},\left|p_{k}\right| \leq q^{-1 / q^{2}},\left|r_{k}\right| \leq q^{-1 / q^{2}}$ for all $k$. 
Theorem 2. Under the above assumptions, the equation (13) possesses solutions of the form

$$
y(t)=c t^{1 / q}+\sum_{n=0}^{\infty} a_{n} t^{q^{n}}, \quad c, a_{n} \in \bar{K}_{c}, c \neq 0,
$$

where the series converges on the open unit disk $|t|<1$.

Proof. For the function (14) we have

$$
\begin{gathered}
d y(t)=c^{1 / q}[-1]^{1 / q} t^{q^{-2}}+\sum_{n=1}^{\infty} a_{n}^{1 / q}[n]^{1 / q} t^{q^{n-1}}, \quad[-1]=x^{1 / q}-x, \\
(y \circ y)(t)=c\left(c t^{1 / q}+\sum_{n=0}^{\infty} a_{n} t^{q^{n}}\right)^{1 / q}+\sum_{n=0}^{\infty} a_{n}\left(c t^{1 / q}+\sum_{m=0}^{\infty} a_{m} t^{q^{m}}\right)^{q^{n}} \\
=c^{1+\frac{1}{q}} t^{q^{-2}}+\left(c a_{0}^{1 / q}+c a_{0}\right) t^{q^{-1}}+\sum_{n=0}^{\infty}\left(c a_{n+1}^{1 / q}+c^{q^{n+1}} a_{n+1}\right) t^{q^{n}}+\sum_{l=0}^{\infty} t^{q^{l}} \sum_{\substack{m+n=l \\
m, n \geq 0}} a_{n} a_{m}^{q^{n}} .
\end{gathered}
$$

Finally,

$$
(P(\tau) y)(t)=\sum_{k=0}^{\infty} p_{k+1} c^{q^{k+1}} t^{q^{k}}+\sum_{l=0}^{\infty} t^{q^{l}} \sum_{\substack{i+j=l \\ i \geq 1, j \geq 0}} p_{i} a_{j}^{q^{i}} .
$$

Comparing the coefficients we find that

$$
\begin{gathered}
c=\lambda^{-1}[-1]^{1 / q}, \quad a_{0}^{1 / q}+a_{0}=0, \\
a_{l+1}^{1 / q}\left([l+1]^{1 / q}-\lambda c\right)-\lambda c^{q^{l+1}} a_{l+1}=\lambda \sum_{\substack{m+n=l \\
m, n \geq 0}} a_{n} a_{m}^{q^{n}}+\sum_{\substack{i+j=l \\
i \geq 1, j \geq 0}} p_{i} a_{j}^{q^{i}}+r_{l}, \quad l \geq 0 .
\end{gathered}
$$

By (15), we have $|c| \geq 1$, and either $a_{0}=0$, or $\left|a_{0}\right|=1$. Next, (16) is a recurrence relation (with an algebraic equation to be solved at each step) giving values of $a_{l}$ for all $l \geq 1$. Let us prove that $\left|a_{j}\right| \leq 1$ for all $j$. Suppose we have proved that for $j \leq l$. It follows from (16) that

$$
\left|a_{l+1}[l+1]-\lambda^{q} c^{q} a_{l+1}-\lambda^{q} c^{q^{l+2}} a_{l+1}^{q}\right| \leq q^{-1 / q} .
$$

Suppose that $\left|a_{l+1}\right|>1$. We have $\lambda^{q} c^{q}=[-1]$, so that $\left|\lambda^{q} c^{q}\right|=q^{-1 / q}$, and since $|[l+1]|=q^{-1}$ and $|c| \geq 1$, we find that

$$
\left|a_{l+1}[l+1]\right|<\left|\lambda^{q} c^{q} a_{l+1}\right|<\left|\lambda^{q} c^{q^{l+2}} a_{l+1}^{q}\right|
$$

Therefore the left-hand side of (17) equals $\left|\lambda^{q} c^{q}\right| \cdot\left|c^{q^{l+1}}\right| \cdot\left|a_{l+1}^{q}\right|>q^{-1 / q}$, and we have come to a contradiction. 


\section{References}

[1] L. Carlitz, On certain functions connected with polynomials in a Galois field, Duke Math. J. 1 (1935), 137-168.

[2] L. Carlitz, Some special functions over $G F(q, x)$, Duke Math. J. 27 (1960), 139-158.

[3] D. Goss, Fourier series, measures, and divided power series in the theory of function fields, K-Theory 1 (1989), 533-555.

[4] I. N. Herstein, Noncommutative Rings, The Carus Math. Monograph No. 15, Math. Assoc. of America, J. Wiley and Sons, 1968.

[5] E. Hille, Lectures on Ordinary Differential Equations, Addison-Wesley, Reading, 1969.

[6] A. N. Kochubei, $\mathbb{F}_{q}$-linear calculus over function fields, J. Number Theory 76 (1999), 281-300

[7] A. N. Kochubei, Differential equations for $\mathbb{F}_{q}$-linear functions, J. Number Theory 83 (2000), 137-154.

[8] A. N. Kochubei, Differential equations for $\mathbb{F}_{q}$-linear functions, II: Regular singularity, Finite Fields Appl. 9 (2003), 250-266.

[9] R. S. Pierce, Associative Algebras, Springer, New York, 1982.

[10] B. Poonen, Fractional power series and pairings on Drinfeld modules, J. Amer. Math. Soc. 9 (1996), 783-812.

[11] D. Thakur, Hypergeometric functions for function fields II, J. Ramanujan Math. Soc. 15 (2000), 43-52.

[12] C. G. Wagner, Linear operators in local fields of prime characteristic, J. Reine Angew. Math. 251 (1971), 153-160. 